\title{
Isotropic dynamical models of NGC 4472 (M 49)
}

\begin{abstract}
S. Samurović ${ }^{1}$
Astronomical Observatory, Volgina 7, 11060 Belgrade, Serbia

e-mail: srdjan@aob.bg.ac.rs

Received 21 December 2011 / Accepted 19 March 2012

\section{ABSTRACT}

Context. The existence of dark matter in some early-type galaxies is well-established. We address one such case and study dynamical models of NGC 4472 by means of using both Newtonian and MOdified Newtonian Dynamics (MOND) approaches with particular emphasis on the latter.

Aims. We use the measurements of the radial velocities of globular clusters in NGC 4472 to test the predictions of dynamical models to fit a high and approximately constant value the velocity dispersion with radius for this galaxy.

Methods. The globular clusters of NGC 4472 are used as a tracer of the galaxy's gravitational potential. We calculate the Jeans equation for both the Newtonian (mass-follows-light and dark-matter models) and the MOND approaches assuming spherical symmetry and isotropic orbits.

Results. We solve the isotropic Jeans equation for a spherical approximation and find, assuming a rotational velocity of $50 \mathrm{~km} \mathrm{~s}^{-1}$, that the isotropic Newtonian mass-follows-light models without dark matter cannot provide successful fits at radii larger than $\sim 2 R_{\mathrm{e}}$ and that in the Newtonian approach dark matter is needed. Of the four different MOND models that we tested, two models can provide a successful fit to the velocity dispersion throughout the whole galaxy without assuming any anisotropy (again assuming a rotational velocity of $50 \mathrm{~km} \mathrm{~s}^{-1}$ ), thus showing that in the case of NGC 4472 MOND can describe the dynamics of a massive early-type galaxy with high values of velocity dispersion.
\end{abstract}

Key words. gravitation - galaxies: elliptical and lenticular, cD - galaxies: kinematics and dynamics - galaxies: halos galaxies: individual: NGC 4472 (M 49)

\section{Introduction}

Although some early-type galaxies appear to lack dark matter (see, for example, the case of our nearest elliptical NGC 5128, see Samurović 2010) for some giant ellipticals all evidence inevitably suggests that they are found in massive dark haloes. One such example is a bright elliptical NGC 4472 (M 49) in the Virgo cluster. Numerous detailed studies of this galaxy (for example, Trinchieri et al. 1986; Loewenstein 1992; Saglia et al. 1993; Kronawitter et al. 2000; Zepf et al. 2000; Côté et al. 2003, hereafter $\mathrm{C} 03$ ) have shown that there appears to be a significant amount of dark matter in NGC 4472.

While the dark matter hypothesis remains the most widely used approach in dynamical modeling of all types of galaxies, MOND (MOdified Newtonian Dynamics) (Milgrom 1983) theory has also been shown to successfully fit the rotation curves of spiral galaxies, although when applied to early-type galaxies the results have been ambiguous. The basic assumption of MOND is that the acceleration due to gravitational force does not simply depend upon the mass $m$ as in the Newtonian approach, but that it has a more complex form, $m / \mu\left(a / a_{0}\right)$, where $a_{0}$ is a universal constant, taken to be $a_{0}=1.35_{-0.42}^{+0.28} \times 10^{-8} \mathrm{~cm} \mathrm{~s}^{-2}$ (Famaey et al. 2007) and $\mu\left(a / a_{0}\right)$ is the MOND interpolating function (see Sect. 3.2 for the details on various interpolating functions used in this paper). For $a \gg a_{0}$, the Newtonian acceleration is restored, whereas for $a \ll a_{0}$ the interpolation function becomes $\mu=a / a_{0}$. To model the dynamics of galaxies, it is well-known that the Jeans equation (for both Newtonian and MOND approaches) is one of the most important tools: the declining trend of the velocity dispersion for some galaxy is interpreted as proof of a constant mass-to-light ratio in a given galaxy, hence a lack of dark matter. The Jeans equation is widely used when one models the dynamics of the early-type galaxies (e.g. Romanowsky et al. 2003; Samurović \& Danziger 2005) and it has been shown in the case of the Milky Way (Samurović \& Lalović 2011) that it can be successfully applied using the MOND approach to spiral systems such as our Galaxy. For ellipticals, the Jeans equation was used by Tiret et al. (2007), who modeled NGC 3379 and found that MOND can reproduce the observations on all scales. For NGC 5128, Samurović (2010) showed that dark matter is not needed out to $6.4 R_{\mathrm{e}}$, where $R_{\mathrm{e}}$ is the effective radius and both Newtonian mass-follows-light and MOND models provide good fits to the observed velocity dispersion. Interestingly, Salinas et al. (2011) found that MOND predictions overestimate the observed velocity dispersion (declining profile) for the field elliptical NGC 7507. On the other hand, Richtler et al. (2008) found that the central galaxy of the Fornax cluster, NGC 1399 needs a hypothetical dark halo. We note that for both NGC 3379 and NGC 5128 the velocity dispersion shows a declining trend, which starts at $\sim 200 \mathrm{~km} \mathrm{~s}^{-1}$ for NGC 3379 and $\sim 150 \mathrm{~km} \mathrm{~s}^{-1}$ for NGC 5128. In addition, Angus et al. (2008) showed that by using the measured velocity distribution of satellite galaxies, obtained for Sloan Digital Sky Survey (SDSS) data, one can successfully model the observed declining line-of-sight velocity dispersions. For NGC 1399, one encounters much higher values of the approximately constant value of velocity dispersion: $\sigma \approx 300 \mathrm{~km} \mathrm{~s}^{-1}$

In this paper, we study the brightest optical member of the Virgo cluster NGC 4472, which is a non-central galaxy (the galaxy at the center of the Virgo cluster is another well-studied 
Table 1. Kinematics data for NGC 4472 for the sample of GCs. Upper part: each bin (except for the outermost one) contains approximately 60 objects. Lower part: each bin has a constant width of $2 \operatorname{arcmin}$ (except for the outermost one for which the width is 4 arcmin).

\begin{tabular}{lcrrrrrr}
\hline \hline$\langle r\rangle$ & $\begin{array}{r}\sigma \\
(\operatorname{arcsec})\end{array}$ & $\begin{array}{r}\text { err_ } \sigma \\
\left(\mathrm{km} \mathrm{s}^{-1}\right) \\
\left(\mathrm{km} \mathrm{s}^{-1}\right)\end{array}$ & $s_{3}$ & err_s & $s_{4}$ & err_s & $N$ \\
$(1)$ & $(2)$ & $(3)$ & $(4)$ & $(5)$ & $(6)$ & $(7)$ & $(8)$ \\
\hline 75 & 326 & 30 & -0.017 & 0.322 & -0.505 & 0.643 & 58 \\
185 & 308 & 27 & 0.087 & 0.304 & -0.010 & 0.030 & 65 \\
260 & 342 & 32 & 0.358 & 0.327 & 0.371 & 0.655 & 56 \\
435 & 320 & 25 & 0.279 & 0.267 & 0.419 & 0.535 & 84 \\
\hline 60 & 318 & 36 & 0.009 & 0.057 & -0.011 & 0.040 & 38 \\
180 & 330 & 24 & 0.010 & 0.036 & -0.009 & 0.025 & 97 \\
300 & 305 & 23 & 0.042 & 0.038 & 0.016 & 0.027 & 85 \\
480 & 355 & 38 & 0.064 & 0.054 & 0.029 & 0.037 & 43 \\
\hline
\end{tabular}

Notes. See text for details. Column (1): central point for a given bin. Column (2): velocity dispersion for the sample of GCs in a given bin. Column (3): formal errors for the velocity dispersion of the sample of GCs in a given bin. Column (4): $s_{3}$ parameter for the sample of GCs in a given bin. Column (5): formal errors for the $s_{3}$ parameter for the sample of GCs in a given bin. Column (6): $s_{4}$ parameter for the sample of GCs in a given bin. Column (7): formal errors for the $s_{4}$ parameter for the sample of GCs in a given bin. Column (8): number of GCs in a given bin.

object, NGC $4486=\mathrm{M} 87$ ). We use the globular cluster (GC) data from C03, who estimate that NGC 4472 contains $\sim 6000$ GCs projected within $100 \mathrm{kpc}$ from its center as tracers of the mass distribution: the velocity dispersion of this galaxy is approximately constant and equal to $\sim 320 \mathrm{~km} \mathrm{~s}^{-1}$ throughout the whole galaxy providing an interesting way of testing the MOND approach on an early-type galaxy with a high fraction of dark matter in the Newtonian approach. One should note that when solving the Jeans equation, the problem of the massanisotropy (Tonry 1983) may occur: for example, unaccountedfor anisotropies in the orbits of the tracers may mimic the existence (or lack) of the dark component. The example of NGC 4472 provides an opportunity to avoid this problem since it is shown that the orbits of GCs in this galaxy are isotropic (see $\mathrm{C} 03$ and also below). Thus, in this paper we study the dynamical isotropic Newtonian and MOND models of NGC 4472, assuming spherical symmetry.

The plan of the paper is as follows: in Sect. 2, we present the observational data related to NGC 4472; in Sect. 3, we solve the isotropic Jeans equation using two Newtonian models (one that takes into account that mass-follows-light and the other dark matter), and the MOND approach (in four different models) and study the cumulative mass and the mass-to-light ratio profiles of NGC 4472, and finally in Sect. 4 we present our conclusions.

\section{Observational data}

The galaxy NGC 4472 (=M 49) is one of the most massive galaxies in the Virgo cluster. In this paper, we adopt the distance of $16.7 \pm 0.6 \mathrm{Mpc}$ (Blakeslee et al. 2009). At this distance, 1 arcsec corresponds to $81 \mathrm{pc}$ and 1 arcmin corresponds to $4.86 \mathrm{kpc}$. The effective radius of NGC 4472 is $R_{\mathrm{e}}=104^{\prime \prime}$ $(=8.42 \mathrm{kpc})$ and is taken from the RC3 catalog (de Vaucouleurs et al. 1991). This galaxy has a low systemic velocity, $v_{\text {gal }}=$ $996 \pm 18 \mathrm{~km} \mathrm{~s}^{-1}$ (HyperLeda database). The total $B$ magnitude is $B_{\mathrm{T}}=9.3$ (according to Blakeslee et al. 2009, based on the NED database). Throughout the paper, the Hubble constant $h_{0}=0.70$ is assumed.

\subsection{Globular clusters of NGC 4472}

We analyzed the GCs data set of C03, which contains 263 GCs extending out to $\sim 570$ arcsec. We present the kinematics of
NGC 4472 based on the GCs in Table 1 and in Fig. 1. The radial velocities of GCs were used to determine the kinematics of NGC 4472: we calculated, using standard definitions, the velocity dispersion, the skewness and kurtosis parameters, $s_{3}$ and $s_{4}$, which describe the asymmetric and symmetric departures from the Gaussian in each bin. Negative values of kurtosis imply that tangential anisotropies dominate and positive values of kurtosis indicate the dominance of radial orbits. We determined the kinematics of NGC 4472 using two different binnings: (a) in the first one, we placed approximately 60 objects in each bin, except for the outermost bin where we have 84 GCs; and (b) in the second one, we kept the width of the each bin constant (two arcmin), again with the exception of the outermost one, which has the width of four arcmin (we grouped here two outermost bins, because in the bin between eight and ten arcmin only 8 GCs are present). As one can see from Table 1 and Fig. 1, both binnings produce very similar results and in our analysis below we concentrate on the binning "(a)" for simplicity but we also address the binning "(b)" when necessary. From Table 1 and Fig. 1, one can see that the profile of the velocity dispersion is approximately constant with a high value $\left(\sigma \approx 320 \mathrm{~km} \mathrm{~s}^{-1}\right)$. One can also see that the values of $s_{4}$ are consistent with zero throughout the whole galaxy in all bins and in all our models isotropy of the orbits will always be assumed $\left(s_{4}=0\right.$, i.e. $\beta_{*}=0$, see below). We note that when all GCs are put into one bin the $s_{4}$ parameter is again consistent with zero: $s_{4}=0.178 \pm 0.302$ (and $s_{3}=0.188 \pm 0.151$ when all GCs are in one bin). This is in agreement with the detailed analysis of $\mathrm{C} 03$, who found that they "...have been able to show by direct means that an isotropic velocity ellipsoid is a highly accurate description of GC kinematics".

The ellipticity of the entire system of GCs is approximately 0.16 (Lee et al. 1998), and in our analysis described below we assumed spherical symmetry. This assumption had been previously applied in models of NGC 4472, such as in Loewenstein (1992), Kronawitter et al. (2000), Zepf et al. (2000), and C03, although as noted by Zepf et al. (2000) this “...is not strictly true for NGC 4472, but it not likely to be too far wrong given that the observed ellipticity of the globular cluster systems is 0.1-0.2 (e.g., Lee et al. 1998)". More specifically, in the case of NGC 4472 we expect that the error in the mass-to-light ratio made using such an assumption is no greater than approximately $10 \%$ as shown in Kronawitter et al. (2000) (Sect. 5.1, where their 


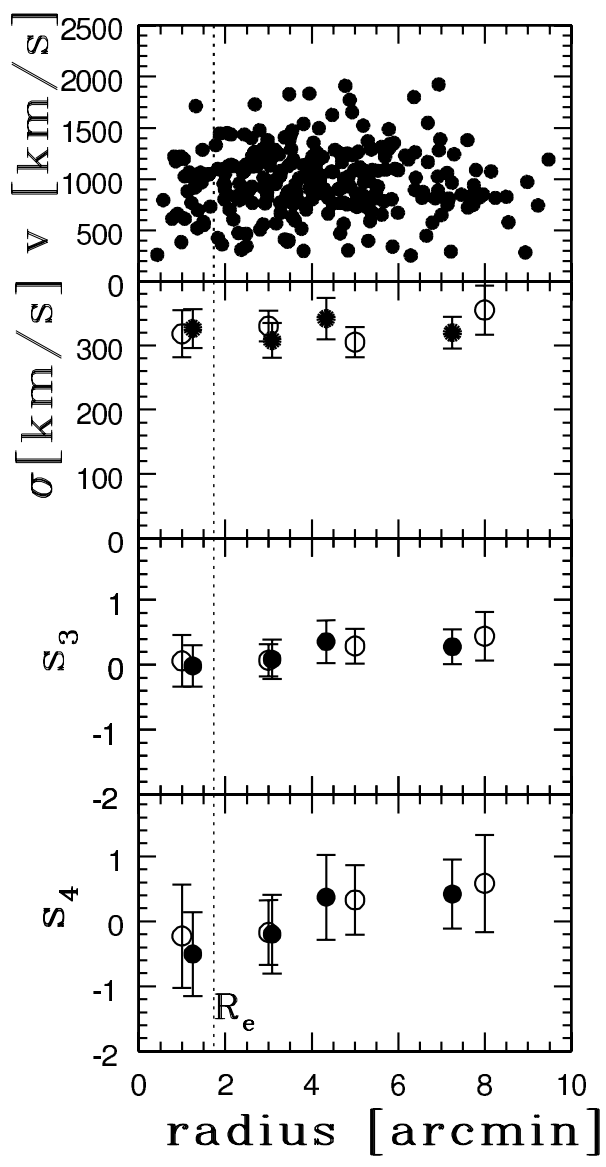

Fig. 1. Kinematics of NGC 4472 based on the sample of the GCs. From top to bottom: radial velocity of the GCs in $\mathrm{km} \mathrm{s}^{-1}$; velocity dispersion in $\mathrm{km} \mathrm{s}^{-1}$ calculated in a given bin and the $s_{3}$ and $s_{4}$ parameters, which describe symmetric and asymmetric departures from the Gaussian, respectively. Two different binnings were used: a) the binning with approximately $60 \mathrm{GCs}$ per bin (except for the outermost one) (full circles) and b) the binning with a fixed width of the bin ( 2 armin, except for the outermost one where width is 4 arcmin) (open circles). See text for details.

estimates of uncertainties due to rotation and non-sphericity are presented).

For the Jeans modeling, we also needed the radial surface density profile of the GCs in NGC 4472. The profile that we used is based on the one presented in Rhode \& Zepf (2001; see their Fig. 5 and Table 2): they fitted the radial surface density (from their Table 2) with the expression $\log N_{\mathrm{cl}}=(1.91 \pm 0.03)-(1.28 \pm$ $0.04) \log r$, where $N_{\mathrm{cl}}$ is the surface density of GCs and $r$ is in arcminutes. They note that this "single power law does not provide a good fit over the entire radial range". This is why we performed a fitting procedure (on the same data) for the region for which we have GC data. The result is given in Fig. 2 and compared with the result of Rhode \& Zepf ("RZ01" in Fig. 2). Our fit, given by $\log N_{\mathrm{cl}}=(1.81 \pm 0.05)-(1.10 \pm 0.04) \log r$, resulted in a smaller $\chi^{2}$ value $\left(\chi^{2}=0.153\right)$ with respect to the fit of Rhode \& Zepf $\left(\chi^{2}=0.765\right)$ and therefore $\alpha=\mathrm{d} \ln \rho / \mathrm{d} \ln r=-2.10$ was used in the models below. As can be seen from Fig. 2, our fit is not radically different from that obtained in Rhode \& Zepf (2001) but in our models we wanted to include a fit that represents as closely as possible the distribution of GCs in the region that we analyze and from Fig. 2 one can see that the innermost region (inside $\sim 1 R_{\mathrm{e}}$ ) is more accurately described by our fit. The quoted value of the fit by Rhode \& Zepf is obtained over the whole radial

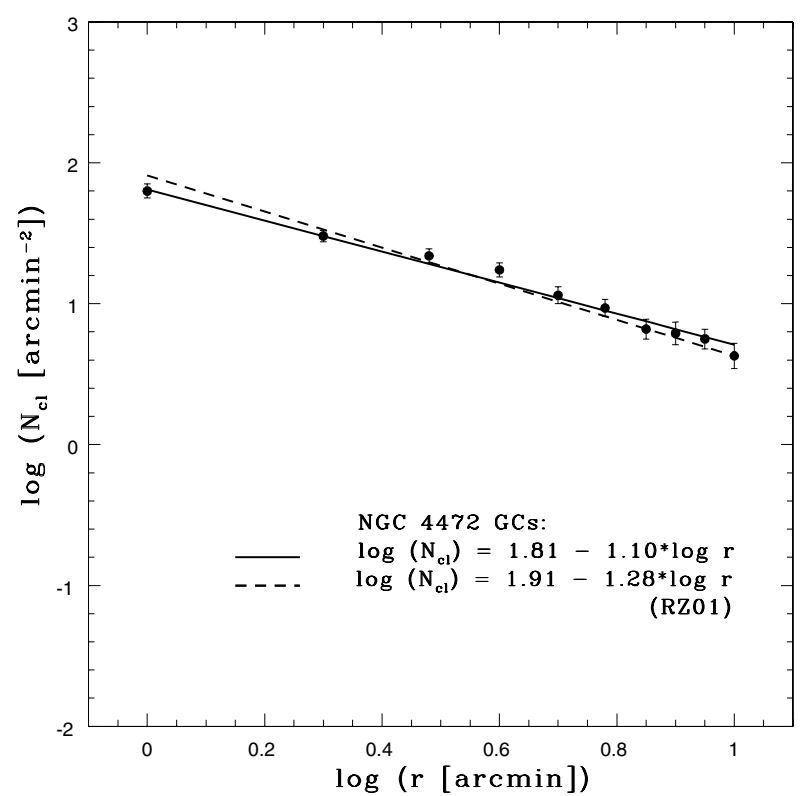

Fig. 2. Radial distribution of GCs in NGC 4472. A power law is fitted to the radial surface density of GCs: $N_{\mathrm{cl}} \propto r^{-\gamma}$ (see text for details). The dashed line is the fit from the paper by Rhode \& Zepf (2001).

range (out to $\sim 23$ arcmin), whereas our fit is performed for the data interior to $\sim 10 \mathrm{arcmin}$.

\subsection{X-ray data for NGC 4472}

The X-rays emission of NGC 4472 has been well-studied since this is one of the most X-ray luminous early-type galaxies in the local Universe (although it is fainter in X-rays than M 87). Irwin \& Sarazin (1996) observed NGC 4472 using the ROSAT HighResolution Imager and determined the temperature profile of this galaxy, obtaining a fit $T(r)=0.93(r / \text { arcmin })^{0.127} \mathrm{keV}$. Kraft et al. (2011) presented their result for a $100 \mathrm{ks}$ XMM-Newton observations of the hot gas in NGC 4472: from their Fig. 9, one can see that the temperature beyond $\sim 5 \mathrm{kpc}$ remains approximately constant, and $T \approx 1 \mathrm{keV}$. Therefore, in our estimates of the mass (and the mass-to-light ratio) based on the X-rays we used $T=1 \mathrm{keV}$.

The total mass of NGC 4472 interior to the radius $r$ based on the X-ray observations was calculated (as described in Sect. 3.3) using the equation of Kim \& Fabbiano (1995)

$M_{\mathrm{T}}=1.8 \times 10^{12}(3 \beta+a)\left(\frac{T}{1 \mathrm{keV}}\right)\left(\frac{r}{1000^{\prime \prime}}\right)\left(\frac{d}{10 \mathrm{Mpc}}\right) M_{\odot}$,

where the exponent $a$ is related to the temperature of the X-ray halo $\left(T \sim r^{-a}\right)$ and in our calculations was taken to be zero for simplicity - this assumption was made because the weak dependence of the temperature on the radius is not expected to influence the estimate of the total mass significantly - and an error in the parameter $a$ of at most $10 \%$ is expected; $\beta$ is the slope used in the analytic King approximation model and is taken to be $\beta=0.43$ (Trinchieri et al. 1986). The mass-to-light ratio in the $B$-band based on the X-rays was calculated as a function of radius $r$ as

$\frac{M_{\mathrm{T}}}{L_{B}}=1.16 \times 10^{-2} 10^{\frac{B}{2.5}}(3 \beta+a)\left(\frac{T}{1 \mathrm{keV}}\right)\left(\frac{r}{1000^{\prime \prime}}\right)\left(\frac{d}{10 \mathrm{Mpc}}\right)^{-1}$, 
where $B$ is the $B$ magnitude of the galaxy inside radius $r$ (Kim $\&$ Fabbiano 1995).

\section{Dynamical models}

For both approaches, we solve the Jeans equation (e.g. Binney $\&$ Tremaine Binney 2008) for a spherical approximation

$\frac{\mathrm{d} \sigma_{r}^{2}}{\mathrm{~d} r}+\sigma_{r}^{2} \frac{\left(2 \beta_{*}+\alpha\right)}{r}=a_{\mathrm{N} ; \mathrm{M}}+\frac{v_{\mathrm{rot}}^{2}}{r}$.

The parameter $a_{\mathrm{N} ; \mathrm{M}}$ is an acceleration term that is different for each approach. In the Newtonian ("N") approach, it is equal to $a_{\mathrm{N}}=-\frac{G M(r)}{r^{2}}$, and for MOND ("M") models, $a_{\mathrm{M}} \mu\left(a_{\mathrm{M}} / a_{0}\right)=a_{\mathrm{N}}$ (in Sect. 3.2, we present the relevant details for the function $\mu$ ). The parameter $\sigma_{r}$ is the radial stellar velocity dispersion and $\alpha=$ $\mathrm{d} \ln \rho / \mathrm{d} \ln r$ is the slope of the tracer surface density $\rho$ as given above; in the models below, we use $\alpha=-2.10$. The rotation speed $v_{\text {rot }}$ of NGC 4472 is taken to be $v_{\text {rot }}=53_{-25}^{+52} \mathrm{~km} \mathrm{~s}^{-1}$ (see C03, Table 3).

One can use a well-known relation to describe the nonspherical nature of the stellar velocity dispersion, where we write $\beta_{*}$ to avoid confusion with the parameter used in Eqs. (1) and (2), given by

$\beta_{*}=1-\frac{\overline{v_{\theta}^{2}}}{\sigma_{r}^{2}}$,

where $\overline{v_{\theta}^{2}}={\overline{v_{\theta}}}^{2}+\sigma_{\theta}^{2}$ and $0<\beta_{*}<1$ means that the orbits are predominantly radial (equivalent to $s_{4}>0$ ), whereas for $-\infty \leq \beta_{*}<0$ the orbits are mostly tangential (equivalent to $s_{4}<0$ ) (Gerhard 1993). As stressed above, the galaxy NGC 4472 represents a case for which isotropic distribution of the orbits is well-established $(\mathrm{C} 03)$ and our estimate of the $s_{4}$ parameter strongly suggests the absence of departures from a Gaussian, i.e. $s_{4} \approx 0$ (Fig. 1 ).

In all models calculated below, we used the projected line-ofsight velocity dispersion (e.g. Binney \& Mamon 1982) given as

$\sigma_{\mathrm{p}}^{2}(R)=\frac{\int_{R}^{r_{t}} \sigma_{r}^{2}(r)\left[1-(R / r)^{2} \beta_{*}\right] \rho(r)\left(r^{2}-R^{2}\right)^{-1 / 2} r \mathrm{~d} r}{\int_{R}^{r_{t}} \rho(r)\left(r^{2}-R^{2}\right)^{-1 / 2} r \mathrm{~d} r}$,

where the truncation radius, $r_{\mathrm{t}}$, extends beyond the observed kinematical point of the largest galactocentric radius.

Cappellari et al. (2006) studied the correlation between mass-to-light ratio and velocity dispersion in the $I$-band in the inner parts where baryonic matter is expected to dominate for the sample of early-type galaxies (NGC 4472 was not included in their sample). They found, using two-integral Jeans and threeintegral Schwarzschild dynamical models, that the expression that holds is

$M / L_{I}=(3.80 \pm 0.14)\left(\frac{\sigma_{\mathrm{e}}}{200 \mathrm{~km} \mathrm{~s}^{-1}}\right)^{0.84 \pm 0.07}$,

where the value of the velocity dispersion is assumed to that evaluated at the effective radius. When we insert $\sigma_{\mathrm{e}}=$ $310 \mathrm{~km} \mathrm{~s}^{-1}$, we obtain $M / L_{I}=5.49 \pm 0.38$. Since for NGC 4472 , $R-I=0.55$ (from the HyperLeda database), the mass-to-light ratio in the $R$-band becomes $M / L_{R}=6.66 \pm 0.46$. This value is somewhat higher than that obtained using the stellar population synthesis (SPS) models of Bell \& de Jong (2001); using Kodama $\&$ Arimoto (1997) models based on the Salpeter initial mass function (IMF), one finds $M / L_{R}=5.42$. Finally, to establish the mass-to-light ratio in the $B$-band, we use $B-R=1.64$ (again from the HyperLeda database) to find $M / L_{B}=11.36 \pm 0.78$. This value is higher than that found by van der Marel (1991), $M / L_{B}=8.31 \pm 0.17$ (converted to the distance used in this paper). We note that from the SPS analysis of Bell \& de Jong (see their Fig. 3) again using Kodama \& Arimoto (1997) models based on the Salpeter IMF, one obtains $M / L_{B}=9.77$, which is very close to the value used in our Jeans models. We find that the Salpeter IMF best explains the modeled $M / L$ ratio (see also the discussion below in Sect. 3.2). In our models below, we always use $M / L_{B}=10.00$, assuming that it includes only a stellar component, unless noted otherwise. In the discussion below (Sect. 3.3), we present several estimates of the mass-to-light ratio of NGC 4472 based on different methodologies.

\subsection{Newtonian models}

First, we develop a constant mass-to-light ratio model and consider that the stellar mass is distributed in the form of the standard Hernquist (1990) profile

$\rho_{\mathrm{H}}(r)=\frac{M_{\mathrm{T}}}{2 \pi} \frac{a}{r} \frac{1}{(r+a)^{3}}$,

which has two parameters of the total mass $M_{\mathrm{T}}$ and scale length, $a$, where $R_{\mathrm{e}}=1.8153 a$. As stated above, we assume isotropy $\left(\beta_{*}=0\right)$ and a constant mass-to-light ratio, $M / L_{B}=10.00$, and the result of this model (using $28<v_{\text {rot }}<105 \mathrm{~km} \mathrm{~s}^{-1}$ ) is given in Fig. 3: the thick solid line is for $v_{\text {rot }}=50 \mathrm{~km} \mathrm{~s}^{-1}$ and the hatched region encompassing this value is determined by $v_{\text {rot }}=28 \mathrm{~km} \mathrm{~s}^{-1}$ (lower limit) and $v_{\text {rot }}=105 \mathrm{~km} \mathrm{~s}^{-1}$ (upper limit). One can see that for this mass-follows-light approximation, the observed velocity dispersion can be fitted interior to $\sim 1 R_{\mathrm{e}}$ and beyond that region dark matter begins to play an important dynamical role.

Next, we create a Newtonian model with dark matter (again assuming $28<v_{\text {rot }}<105 \mathrm{~km} \mathrm{~s}^{-1}$ ). Numerous models are possible (see, for example, Bullock et al. 2001, for various profiles) but here we chose to present the model (again assuming isotropy), which includes the NFW (Navarro et al. 1997) halo with the following fitted parameters: $\rho_{s}=0.13 \pm 0.02 M_{\odot} \mathrm{pc}^{-3}$ and $r_{\mathrm{s}}=144 \pm 5 \operatorname{arcsec}(\approx 11.7 \pm 0.4 \mathrm{kpc})$. This halo is added to a Hernquist spheroid that includes the stellar matter. The de Vaucouleurs profile is a fair representation of the observed stellar distribution in NGC 4472 between 7 arcsec and $\sim 4$ arcmin, where it begins to diverge although not dramatically (see Kim et al. 2000, Fig. 8), hence the use of the Hernquist profile to describe the de Vaucouleurs law is a useful approximation. This model is presented in Fig. 3: the dashed line represents $v_{\text {rot }}=$ $50 \mathrm{~km} \mathrm{~s}^{-1}$ and the hatched region encompassing it is determined as in the constant mass-to-light ratio model above. This model obviously provides a satisfactory fit to the observed velocity dispersion. To fit the outermost point (binning "a", at $\sim 4.2 R_{\mathrm{e}}$ ), one would need $M / L_{B} \approx 20$, which means that interior to that point the contribution of dark matter is equal to that of baryonic matter. The quality of the fits is given in Table 2 . The obtained fitted parameters imply the virial mass of $\sim 2 \times 10^{12} M_{\odot}$ and the high concentration parameter $c=15.5^{1}$ of the NFW model, which agrees with the results of the high-resolution $N$-body simulations of a $\Lambda$ CDM cosmology (Bullock et al. 2001) for haloes at $z=0$.

\footnotetext{
1 This value is high compared to the most recent simulations which use the latest Wilkinson Microwave Anisotropy Probe (WMAP) parameters (e.g. Duffy et al. 2008).
} 


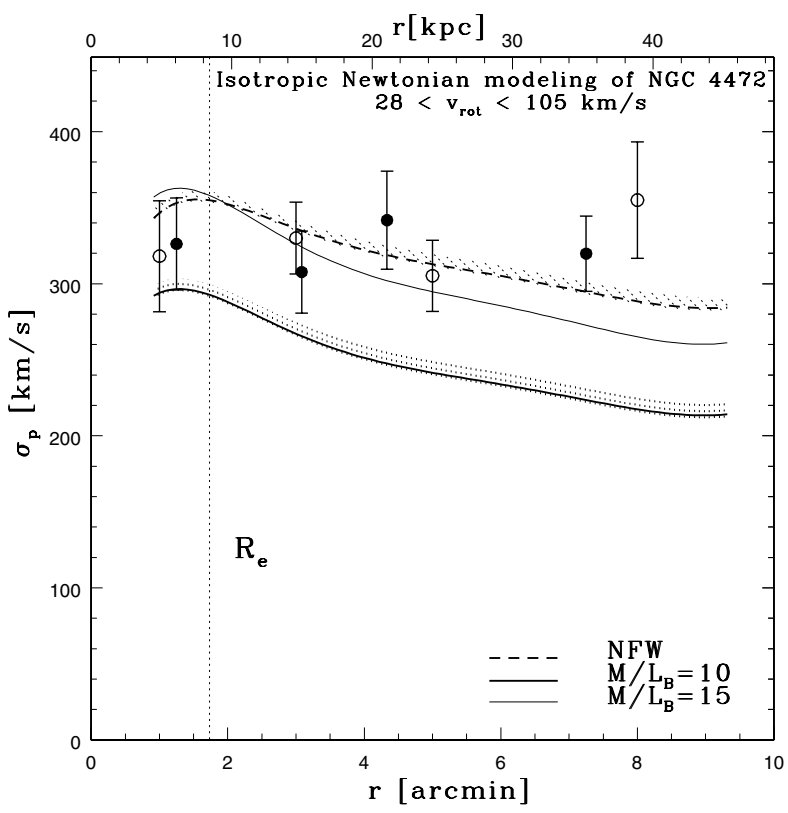

Fig. 3. Isotropic Jeans modeling of the projected velocity dispersion of NGC 4472 using a Newtonian approach: mass-follows-light $\left(M / L_{B}=\right.$ 10) and NFW models are shown. One effective radius is indicated by a vertical dotted line. Solid and dashed lines are plotted using $v_{\text {rot }}=$ $50 \mathrm{~km} \mathrm{~s}^{-1}$. A scaled-up version of the Newtonian mass-follows-light with $M / L_{B}=15$ is plotted with a thin solid line. Two hatched regions (the upper one is for the NFW model and the lower one is the Newtonian constant mass-to-light ratio model with $M / L_{B}=10$ ) for each approach take into account various values of the rotational velocity: the lower limit in both cases is for $v_{\text {rot }}=28 \mathrm{~km} \mathrm{~s}^{-1}$ and the upper limit in both cases is for $v_{\text {rot }}=105 \mathrm{~km} \mathrm{~s}^{-1}$.

We also made a scaled-up version of the Newtonian massfollows-light model with $M / L_{B}=15$, which is plotted with a thin solid line in Fig. 3 and one can see that the quality of this fit is poorer than that of the model with the NFW halo $\left(\chi_{\mathrm{a}}^{2}=\right.$ 5.56 and $\chi_{\mathrm{b}}^{2}=9.49$ for the binnings "a" and " $\mathrm{b}$ " as described earlier). Although high, this value of the mass-to-light ratio is not inconsistent with the PEGASE2 models ${ }^{2}$ with $x=-1.85$ (where $x$ is the logarithmic slope used in the parametrizations of the IMF, see also Sect. 3.2), for which for $B-R \approx 1.6$ one obtains $M / L_{B} \approx 15.8$ (see Bell \& De Jong 2001, Fig. 4).

Taking both Newtonian models into account (and assuming the rotational velocity $28<v_{\text {rot }}<105 \mathrm{~km} \mathrm{~s}^{-1}$ ), one may reach the conclusion that a significant amount of dark matter is indeed needed in NGC 4472, which agrees with the results found in the literature.

\subsection{MOND models}

As mentioned in the introduction, we tested several MOND models using the Jeans equation assuming isotropy, a spherical approximation, and a constant mass-to-light ratio, $M / L_{B}=10$.

The Newtonian acceleration is given as $a_{\mathrm{N}}=a \mu\left(a / a_{0}\right)$, where $a$ is the MOND acceleration, $a_{0}$ is the universal constant given in the introduction, and $\mu(x)$ is the MOND interpolating function that is different for each MOND model. The following MOND models were tested in order to determine

2 PEGASE2 (http://www2.iap.fr/users/fioc/PEGASE.html) is the code used to compute the spectral evolution of galaxies: using this code, one follows the evolution of the stars, gas, and metals for a law of star formation and a stellar IMF. whether an additional dark matter component is needed or MOND alone can correctly describe the dynamics of NGC 4472: (i) the "simple" MOND formula from Famaey \& Binney (2005), (ii) the "standard" formula (Sanders \& McGaugh 2002), (iii) the Bekenstein's "toy" formula (Bekenstein 2004), and (iv) the Zhao (2007) formula. The expressions for the circular velocities for the first three functions are given in Samurović \& Ćirković (2008):

\section{A "simple" MOND formula is given by}

$$
\mu(x)=\frac{x}{1+x} .
$$

2. A "standard" MOND formula is given by

$$
\mu(x)=\frac{x}{\sqrt{1+x^{2}}} .
$$

3. The "toy" MOND model is described with

$$
\mu(x)=\frac{-1+\sqrt{1+4 x}}{1+\sqrt{1+4 x}} .
$$

4. Finally, we analyze the MOND model conceived by Zhao (2007); this model was created to explain the exact amount of dark energy and it belongs to the same family of MOND models as the "simple" model:

$$
\mu(x)=1-\left(1+\frac{x}{n}\right)^{-n} .
$$

While the "simple" model is obtained for $n=1$, the Zhao (2007) model is obtained for $n=3$. Calculation of the circular velocity for $n=3$ is much more complicated and is therefore done numerically.

We again present the models for which the rotational velocity is $28<v_{\text {rot }}<105 \mathrm{~km} \mathrm{~s}^{-1}$. The quality of the fits is given in Table 2 for $v_{\text {rot }}=50 \mathrm{~km} \mathrm{~s}^{-1}$. From Fig. 4 , one can see that all MOND models can provide a successful fit interior to $\sim 2 R_{\mathrm{e}}$. Beyond $\sim 2 R_{\mathrm{e}}$, only the "toy" model can provide a fit $\left(\chi^{2}=1.53\right)^{3}$ to the observed velocity dispersion points, whereas the "simple" MOND model marginally fails to fit the outermost point (for the binning "a", $\chi^{2}=4.06$ ). This is why we decided to present an additional fit of the "simple" model for which we tested a higher value of the mass-to-light ratio while still taking into account Eq. (6) (see also below) of $M / L_{B}=12$. One can see that this higher value of the mass-to-light ratio (assuming no contribution of dark matter) manages to provide a fit to all the observed velocity dispersion points; the obtained fit is, together with the fit obtained using the "toy" MOND model, the closest of all tested MOND models, with $\chi^{2}=1.58$ for the binning " $a$ ". The remaining two MOND models would need to have an anisotropic distribution of the orbits (tangential anisotropies with $\beta_{*} \sim-0.2$, which are however inconsistent with either the available kinematics of NGC 4472 or a higher value of the mass-to-light ratio (implying that there is an additional dark component or the IMF based on the PEGASE2 models with $x=-1.85$, see Bell \& de Jong 2001). In all our models, we set the mass-to-light ratio to 10 in the $B$-band (except for one case, where $M / L_{B}=12$ was taken) and this was the only free parameter in our model. All other quantities entering the Jeans equation were fixed: $\beta_{*}=0$ (isotropy was always

${ }^{3}$ All the quoted $\chi^{2}$ values refer to the binning "a" unless noted otherwise. 
Table 2. Fits for the Newtonian (constant mass-to-light ratio and NFW models) and the MOND isotropic models (assuming $\left.v_{\mathrm{rot}}=50 \mathrm{~km} \mathrm{~s}{ }^{-1}\right)$ for two different binnings ("a" and "b" as described in Sect. 2.1).

\begin{tabular}{lccc}
\hline \hline Model & $M / L_{B}$ & $\chi_{\mathrm{a}}^{2}$ & $\chi_{\mathrm{b}}^{2}$ \\
$(1)$ & $(2)$ & $(3)$ & $(4)$ \\
\hline Newtonian (constant $M / L$ ratio) & 10 & 21.38 & 26.97 \\
Newtonian (NFW) & $20^{*}$ & 2.76 & 5.06 \\
MOND "simple" & 10 & 4.06 & 6.07 \\
MOND "simple" & 12 & 1.58 & 2.65 \\
MOND "standard" & 10 & 12.29 & 15.21 \\
MOND "toy" & 10 & 1.53 & 2.15 \\
MOND "Zhao" & 10 & 8.16 & 10.53 \\
\hline
\end{tabular}

Notes. Column (1): the name of the model. Column (2): mass-to-light ratio in the $B$-band used. ${ }^{*}$ ) For the NFW model, the mass-to-light ratio is given for the outermost point (binning "a"): note also that this value refers to the dynamical mass-to-light ratio, whereas in all other cases in this table the dynamical mass-to-light ratio and the stellar mass-to-light ratio coincide. Column (3): reduced $\chi^{2}$ of the fit for a given model (see text for details).

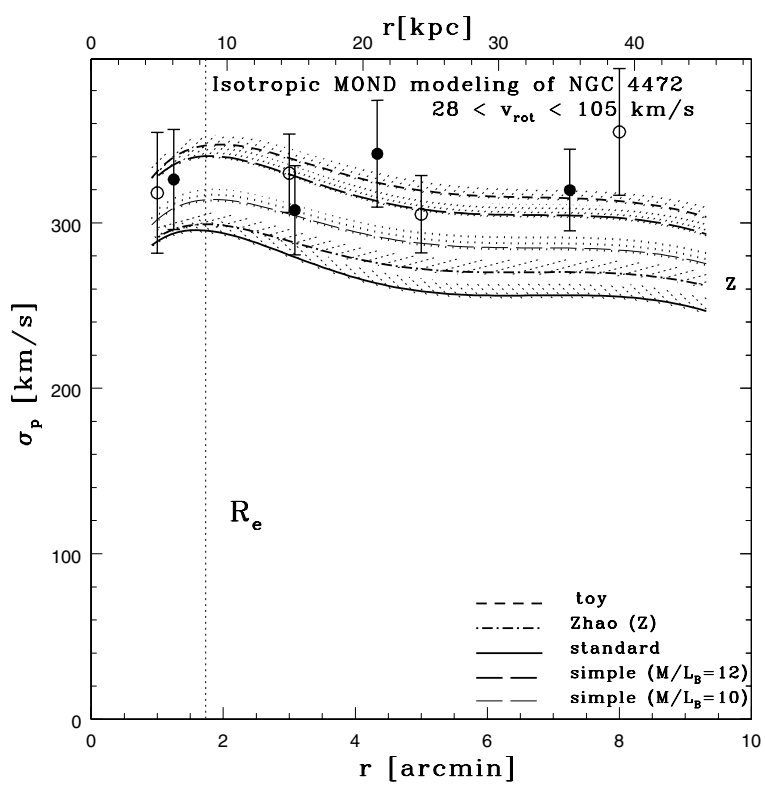

Fig. 4. Isotropic MOND models of the projected velocity dispersion of NGC 4472. One effective radius is indicated by a vertical dotted line. In this figure for all the models different types of lines indicate that the rotational velocity $v_{\text {rot }}=50 \mathrm{~km} \mathrm{~s}^{-1}$ is assumed. The hatched regions are determined by the rotational velocity of $28 \mathrm{~km} \mathrm{~s}^{-1}$ (lower limit) and $105 \mathrm{~km} \mathrm{~s}^{-1}$ (upper limit). To avoid confusion, the Zhao model has been labeled with " $Z$ " on the right hand side of the figure.

assumed), $\alpha=-2.10$, and $28<v_{\text {rot }}<105 \mathrm{~km} \mathrm{~s}^{-1}$. The resulting $\chi^{2}$ values (for $v_{\text {rot }}=50 \mathrm{~km} \mathrm{~s}^{-1}$ ) can be found in Table 2 .

We note that in our MOND models we did not take into account the "external field effect", which has implications for nonisolated systems. We used the results of Richtler et al. (2011), who estimated the external gravitational fields acting on Virgo and Coma galaxies. The galaxy NGC 4472 was on their list and they calculated the value of the external field at the position of this galaxy to be $\sim 0.1 a_{0}$ (see their Fig. 3) hence neglected the influence of this effect because this value was smaller than the values estimated in the inner parts of their galaxies. In a similar manner, we calculated the acceleration in the inner parts of NGC 4472, which we analyzed finding that indeed $a_{0}^{\text {ext }}<a_{0}^{\text {int }}$, where $a_{0}^{\text {int }}$ is the acceleration in the inner parts (interior to $\sim 10 \mathrm{arcmin}$ ) and $a_{0}^{\text {ext }}$ is the acceleration of the external gravitational field acting on NGC 4472.

\subsection{The cumulative mass and the mass-to-light ratio of NGC 4472}

We now present the estimates of the cumulative mass and the mass-to-light ratio based on various methodologies. The results are given in Fig. 5, where we provide the results based on two Newtonian estimates, namely one for X-ray data and the so-called "tracers mass estimator" (TME; Evans et al. 2003), and four MOND formulas are given. The estimates based on $\mathrm{X}$-rays are derived from Eqs. (1) and (2). The TME methodology enables one to estimate the enclosed mass from the projected positions and the line-of-sight velocities of a given tracer population (such as GCs in the case of NGC 4472). The usual assumptions are that the tracer population is spherically symmetric and has a number density that obeys a power law, $\rho(r)=\rho_{0}(a / r)^{\gamma}$, where $a$ is constant and $\gamma$ is a parameter determined from the surface density of the tracer population (see details and comparisons with other techniques in e.g. Samurović 2007, Chap. 5), and these assumptions were adopted in the present work. The advantage of this method is independent method can be used to establish the total galaxy mass for various galaxies at large radii, and the disadvantage is the small number of systems that have been studied in this way so far. Additional observations of various tracers for a larger sample of early-type galaxies will provide the opportunity to compare their independent estimates of cumulative masses. At $\sim 7$ arcmin (the outermost velocity dispersion point), one can see that the mass-to-light ratio is $M / L_{B} \sim 20$ in the Newtonian TME model, which is consistent with the result found in the Jeans modeling and shown in Fig. 3 and Table 2 (assuming rotational velocity $28<v_{\text {rot }}<105 \mathrm{~km} \mathrm{~s}^{-1}$ ).

In Fig. 5 , one can see that interior to $\sim 6$ arcmin there is a discrepancy between the X-ray and TME masses, which however decreases in the outermost regions (10 arcmin). The reason for this discrepancy might be a lack of hydrostatic equilibrium in NGC 4472: for an example of this we refer by Diehl \& Statler (2007), who tabulate the position angles in the optical and X-ray domains, $\mathrm{PA}_{\mathrm{opt}}=160 \pm 0.5$ and $\mathrm{PA}_{\mathrm{X}}=136 \pm 13$ degrees, respectively. This discrepancy warrants a further study. The X-ray mass estimate at $\sim 6$ arcmin is much lower than that based on the TME methodology and does not suggest the existence of dark matter within this radius (inferred using the Jeans equation). Thus, from our Newtonian approach one may conclude that the TME methodology provides a more accurate estimate of the cumulative mass of NGC 4472.

Using both Newtonian estimates of the cumulative mass (TME and X-rays), the overall conclusion is that the estimated 


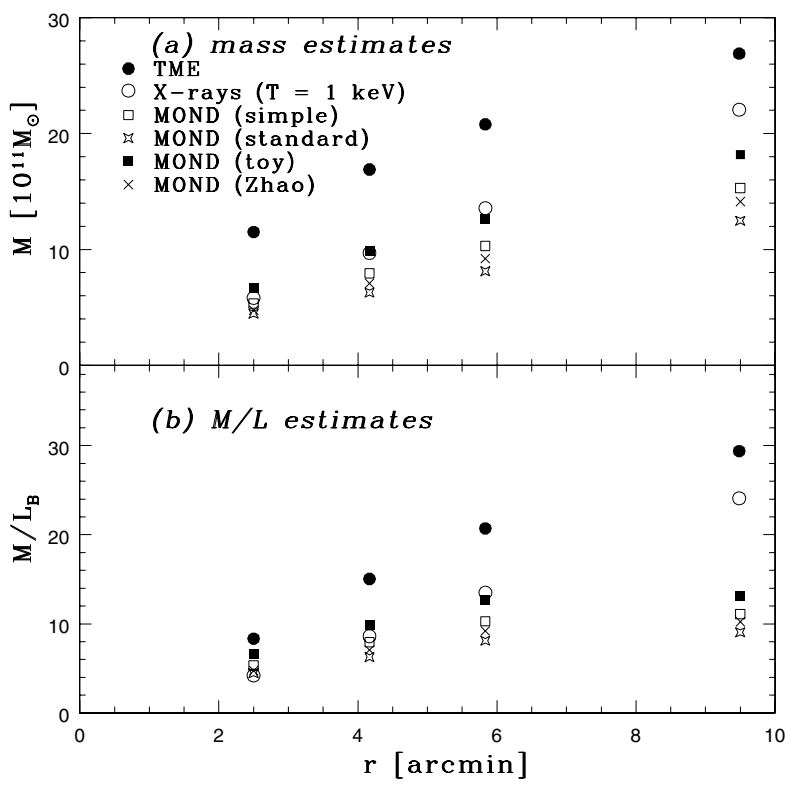

Fig. 5. Cumulative Newtonian and MOND mass (upper panel) and Newtonian and MOND mass-to-light ratio (lower panel). In the Newtonian approach we used the X-rays with temperature $T=1 \mathrm{keV}$ and the "tracer mass estimator" (TME). In the MOND approach we tested four different models: "simple", "standard", Bekenstein's "toy" and Zhao model (see text for details).

mass (and the mass-to-light ratio) clearly indicates that dark matter exists in the outermost regions. On the other hand, the predictions of various MOND formulas indicate that the mass and the mass-to-light ratio are consistent with the stellar population only throughout the whole galaxy. In Fig. 5, the estimates of the mass (and the mass-to-light ratio) based on the MOND models used in this paper are also given. The masses were calculated using the circular velocities of each model. All mass-to-light ratios of the MOND models tested in this paper tend to group, in the outermost region, around $M / L_{B} \sim 10$, except for the "toy" model, which has a somewhat higher value, $M / L_{B} \sim 13$. The results of the Jeans modeling presented in Fig. 4 imply that the "simple" MOND model with $10<M / L_{B}<12$ together with the "toy" model with $M / L_{B}=10$ provide the best fits of all tested MOND models to the observed velocity dispersion (assuming the rotational velocity of $50 \mathrm{~km} \mathrm{~s}^{-1}$ ). These values of the mass-to-light ratio are consistent with (a) the SPS analysis shown above and (b) the estimate obtained by calculating the cumulative mass-to-light ratio of NGC 4472 using the MOND formulas (shown in Fig. 5). A detailed analysis of the stellar populations in NGC 4472 will provide an insight into the stellar mass-to-light ratio of this galaxy and help us to disentangle the contribution of dark matter and thus to more tightly constrain the parameters necessary for the analysis of various MOND models (Samurović et al., in prep.).

\section{Conclusions}

We have studied the dynamics of the early-type galaxy NGC 4472 using the spherical Jeans equation assuming isotropy: the velocity dispersion out to $\sim 7 \operatorname{arcmin}\left(=4.2 R_{\mathrm{e}}\right)$ was modeled in order to show whether the MOND theory can provide a successful fit to the observed data. Our conclusions are as follows:

1. The kinematics of NGC 4472 were studied and the velocity dispersion was found to remain approximately constant at $320 \mathrm{~km} \mathrm{~s}^{-1}$ throughout the whole galaxy. We calculated both skewness and kurtosis, which are both consistent with zero implying that the orbits of GCs in NGC 4472 are close to isotropic. In all of our dynamical models, we assumed isotropy to avoid the mass-anisotropy degeneracy which may lead to incorrect conclusions about the existence of dark matter (and the validity of the MOND theory) in early-type galaxies.

2. We solved the Jeans equation for two isotropic Newtonian models: mass-follows-light and NFW models, both in the spherical approximation. We found that NGC 4472 indeed contains a significant amount of dark matter: interior to $\sim 4.2 R_{\mathrm{e}}$, the contribution of dark matter is approximately equal to that of baryonic matter. To fit the outermost radial point, a high mass-to-light ratio of $M / L_{B} \approx 20$ is needed. Using $M / L_{B}=10$ based only on the baryonic matter (and in agreement with the SPS models), one can fit only the inner region of NGC $4472\left(\sim 1 R_{\mathrm{e}}\right)$.

3 . We also solved the Jeans equation for a spherical approximation for four different MOND models, again assuming isotropy. We found (assuming a rotational velocity $28<$ $v_{\text {rot }}<105 \mathrm{~km} \mathrm{~s}^{-1}$ ) that the "simple" (with $10<M / L_{B}<12$ ) and "toy" (with $M / L_{B}=10$ ) models can provide successful fits to the observed velocity dispersion, and we conclude that these models are consistent with a no dark-matter hypothesis. Two other tested MOND models, the "standard" and Zhao (2007) models could provide a fit to the observed velocity dispersion interior to $\sim 2 R_{\mathrm{e}}$, whereas in the outer parts one would need to invoke anisotropies and/or additional dark matter. The future observations of tracers such as GCs in NGC 4472 will hopefully determine more precisely and to larger radii the dispersion and the rotational velocity in this galaxy thus providing a possibility of more tightly constraining dynamical models of this early-type galaxy.

Acknowledgements. This work was supported by the Ministry of Education and Science of the Republic of Serbia through the project No. 176021, "Visible and Invisible Matter in Nearby Galaxies: Theory and Observations". This research made use of the NASA/IPAC Extragalactic Database (NED), which is operated by the Jet Propulsion Laboratory, California Institute of Technology, under contract with the National Aeronautics and Space Administration. We acknowledge the usage of the HyperLeda database (http://leda.univ-lyon1.fr). The author gratefully acknowledges the valuable comments of the referee, which helped to significantly improve the quality of the manuscript and correct the errors present in its initial version.

\section{References}

Angus, G. W., Famaey, B., Tiret, O., Combes, F., \& Zhao, H.S 2008, MNRAS, 383, L1

Bell, E. F., \& de Jong, R. S. 2001, ApJ, 550, 212

Bekenstein, J. 2004, Phys. Rev. D, 70, 083509

Binney, J. J., \& Mamon, G. 1982, MNRAS, 200, 361

Binney, J. J., \& Tremaine, S. 2008, Galactic Dynamics, second Edition (Princeton: Princeton Univ. Press)

Blakeslee, J. P., Jordán, A., Mei, S., et al. 2009, ApJ, 694, 556

Bullock, J. S., Kolatt, T. S., Sigad, Y., et al. 2001, MNRAS, 321, 559

Cappellari, M., Bacon, R., Bureau, M., et al. 2006, MNRAS, 366, 1126

Côté, P., McLaughlin, D. E., Cohen, J. G., \& Blakeslee, J. P. 2003, ApJ, 591, 850 (C03)

de Vaucouleurs, G., de Vaucouleurs, A., Corwin, H. G. Jr., et al. 1991, Third Reference Catalogue of Bright Galaxies (New York: Springer-Verlag)

Diehl, S., \& Statler, T. S. 2007, ApJ, 668, 150

Duffy, A. R., Schaye, J., Kay, S. T., \& Dalla Vechia, C. 2008, MNRAS, 390, L64

Evans N. W., Wilkinson M. I., Perrett K. M., \& Bridges T. J. 2003, ApJ, 583, 752

Famaey, B., \& Binney, J. 2005, MNRAS, 363, 603

Famaey, B., Gentile, G., Bruneton, J.-P., \& Zhao, H. S. 2007, Phys. Rev. D, 75, 063002

Gerhard, O. 1993, MNRAS, 265, 213

Hernquist, L. 1990, ApJ, 356, 359 
A\&A 541, A138 (2012)

Irwin, J. A., \& Sarazin, C. L. 1996, ApJ, 471, 683

Kim, D.-W., \& Fabbiano, G. 1995, ApJ, 441, 182

Kim, E., Lee, M. G., \& Geisler, D. 2000, MNRAS, 314, 307

Kodama, T., \& Arimoto, N. 1997, A\&A, 320, 41

Kraft, R. P., Forman, W. R., Jones, C., et al. 2011, ApJ, 727, 41

Kronawitter, A., Saglia, R. P., Gerhard, O., \& Bender, R. 2000, A\&AS, 144, 53

Lee, M. G., Kim, E., \& Geisler, D. 1998, AJ, 115, 947

Loewenstein, M. 1992, ApJ, 384, 474

Milgrom, M. 1983, ApJ, 270, 365

Navarro, J. F., Frenk, C. S., \& White, S. D. M. 1997, ApJ, 490, 493

Rhode, K. L., \& Zepf, S. E. 2001, AJ, 121, 210

Richtler, T., Schuberth, Y., Hilker, M., et al. 2008, A\&A, 478, L23

Richtler, T., Famaey, B., Gentile, G., \& Schuberth, Y. 2011, A\&A, 531, A100

Romanowsky, A. J., Douglas, N. G., Arnaboldi, M., et al. 2003, Science, 5640, 1696

Saglia, R. P., Bertin, G., Bertola, F., et al. 1993, ApJ, 403, 567
Salinas, R., Richtler, T., Bassino, L. P., Romanowsky, A. J., \& Schuberth, Y 2012, A\&A, 538, A87

Samurović S. 2007, Dark Matter in Elliptical Galaxies, Publications of the Astronomical Observatory of Belgrade, 81

Samurović, S. 2010, A\&A, 514, A95

Samurović, S., \& Ćirković M. M. 2008, A\&A, 488, 873

Samurović, S., \& Danziger, I. J. 2005, MNRAS, 363, 769

Samurović, S., \& Lalović, A. 2011, A\&A, 531, A82

Sanders, R. H., \& McGaugh, S. 2002, ARA\&A, 40, 263

Tiret, O., Combes, F., Angus, G. W., Famaey, B., \& Zhao, H. S. 2007, A\&A, 476, L1

Tonry, J. L. 1983, ApJ, 266, 58

Trinchieri, G., Fabbiano, G., \& Canizares, C. R. 1986, ApJ, 310, 637

van der Marel, R. P. 1991, MNRAS, 253, 710

Zepf, S. E., Beasley, M. A., Bridges, T. J., et al. 2000, AJ, 120, 2928

Zhao, H. S. 2007, ApJ, 671, L1 